\title{
Untersuchungen zu Verschleißschutzschichten aus einem Hartstoffsystem in Verbindung mit einer metallischen Matrix
}

\author{
Armin Abel
}

Zum Herstellen hochdichter und haftfester Schichten, d.h. Schichten für hochbeanspruchte Bauteile, eignen sich thermische Spritzverfahren, insbesondere das Hochgeschwindigkeitsflammspritzen (HVOF). Diese Spritzschichten basieren auf unterschiedliche Hartstoffsysteme, wie WC/Co oder $\mathrm{Cr}_{3} \mathrm{C}_{2} / \mathrm{NiCr}$.

Um technologische und wirtschaftliche Vorteile zu erlangen, wurden neuartige Hartstoffsysteme, wie Wolframkarbid-Kobalt-Chrom in Verbindung mit einer ledeburitischen Matrix und Siliziumkarbid in Verbindung mit einer metallischen Matrix, im Rahmen einer Diplomarbeit [1] untersucht. Dabei soll die Härte, die Porosität und die Schichtstruktur der gespritzten Proben und deren signifikante Einflußfaktoren bestimmt werden.

\section{Grundlagen}

\section{Aufbau einer Spritzschicht}

Alle thermisch gespritzten Schichten entstehen aus schmelzflüssigen Metallteilchen, die im hocherhitzten Zustand einen Weg zurücklegen, auf dem sie verschiedenen Gasen und der umgebenden Atmosphäre ausgesetzt sind. Der Sauerstoff besitzt hierbei den größten Einfluß, weshalb sich die Spritzteilchen fast ausnahmslos mit einem Oxidfilm umgeben. Dieser noch weiche oder flüssige Oxidfilm ist einerseits eine Komponente des komplizierten Haftmechanismus und andererseits auch ein Härtebildner, der für die häufig größere Mikrohärte der Spritzschichten von Bedeutung ist.

Wichtig für den Aufbau der Schicht ist die Aufschlaggeschwindigkeit, mit der die Spritzteilchen auftreffen. Je höher die kinetische Energie, desto stärker werden die Tropfen in die Kraterlandschaft der vorbereiteten Oberfläche und die bereits aufgebaute Schicht hineingeschossen. Sie verflachen und gehen mit anderen Teilchen Bindungen ein, die mit Diffundieren, Verschweißen, Verkrallen, Zerplatzen und Versintern erklärt werden.

\section{Vor- und Nachteile des thermischen Spritzens}

Die Vorteile der thermischen Beschichtung haben ihre Ursache in der breiten Anwendungsvielfalt dieses Verfahrens. Es können fast alle Substratwerkstoffe beschichtet und ihre Schichtdicke auf hundertstel Millimeter festgelegt werden. Auch die Vielfalt der erzeugbaren Schichten, mit ihren jeweiligen spezifischen Eigenschaften, lassen viele Anwendungsmöglichkeiten in fast allen Industriezweigen zu.

Häufige Anwendungsmöglichkeiten sind z. B. der Korrosionsschutz, der Verschleißschutz und die Wärme- dämmung. Der Einsatz von thermischen Spritzverfahren kann für die Neufertigung oder die Instandsetzung von Bauteilen verwendet werden. Auch die große Palette an einsetzbaren Verfahren ermöglicht ein breites Anwendungsfeld.

Nachteilig auf die Eigenschaften einer Spritzschicht wirkt sich der heterogene und anisotrope Aufbau der Schicht und deren Mikroporosität aus. Bei bestimmten Substratoberflächen treten außerdem Haftprobleme auf. Belastungen in Form von Torsion, Biegung und Schlag führen bei Spritzschichten zu Ablösungen.

Verfahrensprinzip und charakteristische Merkmale des Hochgeschwindigkeitsflammspritzen (HVOF)

Das HVOF (high velocity oxyfuel flamespraying) zählt zu den jüngeren Verfahren des thermischen Spritzens, die zu einer weiteren Verbesserung der Schichtqualität und zur Erschließung neuer Anwendungsgebiete beigetragen haben. Die Entwicklung dieses Verfahrens ist vor allem auf die Erkenntnis zurückzufuihren, daß beim thermischen Spritzen oftmals die Partikelgeschwindigkeit einen großen Einfluß auf die Schichteigenschaften besitzt. Es stellt eine wertvolle Ergänzung zu den konventionellen thermischen Spritzverfahren dar. Der Einsatz beschränkt sich zwar derzeit im wesentlichen noch auf Spritzwerkstoffe, die auch mit anderen Verfahren aufgebracht werden können, jedoch sind bezüglich der Schichtqualität (Haftung, Dichte, Porosität, Oxidgehalt usw.) zum Teil deutliche Vorteile auszumachen.

Das Prinzip des HVOF besteht darin, einen mit Überschallgeschwindigkeit ausströmenden Gasstrahl hoher Temperatur (ca. 2500 bis $3200^{\circ} \mathrm{C}$ ) zum An- und Aufschmelzen sowie zum Beschleunigen des pulverförmigen Spritzwerkstoffes zu nutzen. Die Aufprallgeschwindigkeiten der Teilchen auf das Substrat sind beim HVOF um ein Vielfaches größer als beim konventionellen Flammspritzen und je nach dem zu verarbeitenden Spritzpulver noch fast doppelt so hoch wie beim atmosphärischen Plasmaspritzen. Die mit einem kontinuierlichen Partikelstrahl erzielten hohen Aufprallgeschwindigkeiten ermöglichen das Herstellen von dichten Schichten mit hohen Haftfestigkeiten.

Charakteristisches Merkmal eines Brenners zum Hochgeschwindigkeitsflammspritzen, im Vergleich mit einem konventionellen Flammspritzbrenner, ist die verlängerte Expansionsdüse. Durch die damit vergrößerte Reaktionszone für das Brenngas und dem Sauerstoff, sowie durch die höheren Gasvordrücke und Gasdurchsätze werden wesentlich höhere Gasgeschwindigkeiten und ein stärker gebündelter Spritzstrahl erreicht. 
Ein weiterer Vorteil des HVOF liegt neben den hohen Partikelgeschwindigkeiten in der besser zu kontrollierenden Erwärmung der Partikel. Dadurch wird der Spritzwerkstoff in seiner Mikrostruktur weniger verändert und thermisch aktivierte Vorgänge, die sich nachteilig auf die Eigenschaften der Schichten auswirken könnten, werden vermieden.

\section{Einflußfaktoren der thermischen Spritzschichten}

Untersuchungen über die Einflüsse beim thermischen Spritzen haben gezeigt, daß sich über 60 verschiedene Einflußgrößen ergeben können, die sich teilweise auch gegenseitig beeinflussen. Im folgenden sollen nur einige Beeinflussungen von Parametern erläutert werden, die bei allen thermischen Spritzverfahren anzutreffen sind.

Um einen optimalen Schichtaufbau zu erreichen, müssen alle Spritzteilchen im schmelzflüssigen Zustand auf den Grundwerkstoff auftreffen. Dieses bedeutet, daß alle Teilchen beim Durchgang durch die Flamme aufgeschmolzen werden und der Wärmeverlust durch die Flugphase nur soweit reduziert wird, daß ein Auftreffen im schmelzflüssigen Zustand möglich ist.

Daher ist auch die Wahl der richtigen Korngrößen von Spritzpulvern wichtig. Mit zunehmenden Durchmesser der Partikel nimmt die Porosität der Schicht zu, wodurch die Haftfestigkeit herabgesetzt wird, anderseits aber eine höhere Elastizität der Spritzschichten zu beobachten ist. Im Gegensatz dazu bewirken Pulver mit kleineren Korngrößen Schichten mit größerer Dichte, härterer Oberfläche und guter Wärmeleitfähigkeit.

Eine weitere wichtige Größe zum Erzielen einer guten Schichtqualität ist der Aufschmelzgrad der Spritzpartikel. Dieser wird durch mehrere Faktoren beeinflußt, wie z. B. durch die Verweilzeit der Partikel in der Flamme, die Flammentemperatur, die Wärmeleitfähigkeit der Partikel und die Strahlungsabsorption.

Die Haftung der Spritzschicht hängt von der kinetische Energie und der Temperatur der Teilchen in Abhängigkeit von der Korngröße und somit vom Verformungsgrad ab. So ist beispielsweise erwiesen, daß ein Vorwärmen des Grundwerkstoffes, d. h. aktivieren der Oberfläche, bis $300^{\circ} \mathrm{C}$ die Haftfähigkeit erhöht und die Oberflächenfeuchtigkeit entfernt. Darüber hinausgehende Vorwärmtemperaturen führen zur verstärkter Oxydation und somit zu einer schlechteren Haftung der Schicht. Durch weitere Vorbehandlungen des Grundwerkstoffes, wie das Strahlen der Oberfläche und das Entfetten durch Ethanol, wird eine gute Haftgrundlage erreicht.

Die Haftfestigkeit der Schicht nimmt beim thermischen Spritzen mit zunehmender Schichtdicke ab. Der Grund dafür sind zunehmende Eigenspannungen in der Spritzschicht, die beim Abkühlen entstehen. Diese Spannungen können Zug- oder Druckspannungen sein, deren Betrag vom Temperaturgradienten und von der Spannungsverteilung in der Schicht abhängig ist.

Als wichtiger Einflußparameter beim HVOF gilt der Spritzabstand, der den senkrechten Abstand zwischen der Brennervorderkante und der Werkstückoberfläche beschreibt. Er steht im direkten Zusammenhang mit dem erreichbaren Aufschmelzgrad und beeinflußt dadurch ebenfalls die zu erwartende Porosität, Verdichtung, Rauhigkeit und den Oxidgehalt.

Die Spritzgeschwindigkeit beeinflußt die zu erwartende Schichtdicke und die Temperatur des Grundwerkstoffes und somit auch die Haftfestigkeit der Schicht.

Im direkten Zusammenhang mit der Spritzgeschwindigkeit ist auch der Vorschub als Einflußgröße beim Hochgeschwindigkeitsflammspritzen von Bedeutung. Definiert wird der Vorschub als der Weg, den der Brenner von Spur zu Spur zurücklegt. Ebenso wie der Vorschub beeinflußt auch die Zahl der Überläufe die Schichteigenschaften. Beide Größen stehen über die Temperatur des Grundwerkstoffes im indirekten Zusammenhang mit der zu erwartenden Dicke und Porosität der Schicht.

Größere Bedeutung beim Hochgeschwindigkeitsflammspritzen kommt auch der Kühlung der zu beschichtenden Werkstücke während des Spritzprozesses zu. Dieses ist besonders wichtig, wenn temperaturempfindliche Materialien verarbeitet werden. Dazu zählen unter anderem auch die zu untersuchten karbidischen Spritzzusätze. Die Kühlung trägt dazu bei, daß die einzelnen Spuren und Lagen der entstandenen Schicht gleichmäßig abkühlen und somit Eigenspannungen in der Schicht vermieden werden. Die Kühlung kann mit Luft oder Kohlendioxid erfolgen.

Ein weiterer Einflußfaktor ist die Pulverförderrate, d.h. der Massenstrom des Spritzzusatzes, der in einer bestimmten Zeit durch die Brennereinheit strömt. Sie ist abhängig von dem eingestellten Trägergasdruck und beeinflußt den Aufschmelzgrad der Spritzpartikel und somit auch die Verdichtung und Porosität der Schicht. Ein weiterer Effekt ist die Erhöhung der Schichtdicke. Ein direkter Einfluß auf den Aufschmelzgrad besitzt das Brenngas-Sauerstoffverhältnis, auch Ratio oder Flammenverhältnis genannt. Es stellt das Mengenverhältnis bei der Verbrennung von Brenngas (z. B. Wasserstoff) und Sauerstoff in der Brennerdüse dar. Das Arbeiten mit einen dem stöchiometrischen Verhältnis nahe gelegenen Brenngasverhältnis führt dazu, daß dem Spritzzusatz eine sehr hohe thermische Energie zukommt, was einen erhöhten Aufschmelzgrad des Spritzzusatzes, aber auch eine höhere Oxidation zur Folge hat. Alle beschriebenen Einflußfaktoren stehen untereinander in Wechselwirkung.

\section{Untersuchungen zur Herstellung von Verschleißschutzschichten}

\section{Verschleißschutzschicht aus Wolframkarbid-Kobalt-} Chrom mit einer ledeburitischen Matrix

Die Ziele der Experimente bestanden darin, ein in der Praxis bereits bewährtes karbidisches Hartstoffsystem in Verbindung mit einer ledeburitischen Matrix zur Erzeugung einer Verschleißschutzschicht mit verbesser- 
ter Korrosionsbeständigkeit zu verwenden. Es soll untersucht werden, ob die neuartige ledeburitische Matrix in Verbindung mit Wolframkarbid-Kobalt-Chrom spritztechnisch verarbeitbar ist.

Zur Herstellung des neuen Spritzzusatzes wird der Werkstoff Amdry 5843 (Firmenbezeichnung) mit einem ledeburitischen Stahlwerkstoff mechanisch gemischt. Das Pulver Amdry 5843, das fast ausschließlich für das HVOF eingesetzt wird, besteht aus Wolframkarbid ( 83 bis $90 \%$ ), Kobalt ( 7 bis $11 \%$ ) und aus Chrom (3 bis $6 \%$ ). Die Eigenschaften der mittels Amdry 5843-Pulvers thermisch gespritzten Schicht weisen eine sehr hohe Verdichtung und somit eine geringe Porosität, eine hohe Härte, geringe Oberflächenrauhigkeit, eine gute Bindung sowie ausgezeichnete Abrasions- und Erosionsbeständigkeit auf. Die Einsatzmöglichkeit dieser Schichten ist begrenzt auf eine Temperatur von ca. $500^{\circ} \mathrm{C}$, die aus der geringeren Oxidationsbeständigkeit des Wolframkarbides resultiert. Durch die Verwendung von Chrom in der Matrix weist die Schicht eine gute Korrosionsbeständigkeit auf.

Das zum Einsatz kommende ledeburitische Stahlpulver (Fe22,5Cr) besitzt einen Anteil von 22,5\% Chrom, ca. $2 \%$ Kohlenstoff und ca. $75 \%$ Eisen. Der hohe Kohlenstoff- und Chromgehalt weist darauf hin, daß es sich hierbei um eine Stahllegierung handelt, in deren Gefüge sich Eisen- und Chromkarbide befinden. Diese Stähle sind im Gegensatz zu reinen ledeburitischen Fe-CLegierungen duktil. Sie besitzen durch die Karbidbestandteile eine gute Verschleißbeständigkeit, bei entsprechenden Chromanteilen auch gute Korrosionseigenschaften. Die Kornfraktion des Pulvers beträgt 53 bis $90 \mathrm{~mm}$.

Über die Eigenschaften dieses ledeburitischen Werkstoffes in einer hochgeschwindigkeitsflammgespritzten Schicht ist bis zur Zeit nichts oder nur sehr wenig bekannt. Für die durchgeführten Spritzversuche lagen auch keinerlei Kenntnisse über eine optimale Parameterkombination für den Spritzvorgang vor.

Es wurden die Versuche nach einer statistischen Versuchsplanung nach TAGUOHI ausgeführt.

\section{Auswahl der zu untersuchenden Parameter}

Die Auswahl der zu untersuchenden Parameter wurden in den Experimenten auf fünf beschränkt, da aus den praktischen Erfahrungen früherer Experimente diese die Haupteinflüsse darstellen.

Die ausgewählten Untersuchungsparameter, wie schon beschrieben, sind der Spritzabstand, das Mischungsverhältnis der Pulver, die Pulverförderrate, das Brenngasoder auch Flammenverhältnis und die Schritthöhe (Vorschub).

\section{Versuchsdurchführung und -auswertung}

In Vorbereitung auf die Versuchsdurchführung wurden die Massenanteile der beiden Pulversorten ausgewogen, miteinander vermischt und getrocknet.

Als Trägerwerkstoff wurden Flachproben benutzt,die mit Ethanol im Ultraschallbad entfettet, mit Korund gestrahlt und nochmals im Ultraschallbad entfettet wurden.

Vor dem Spritzen wurde die Probenoberfläche aktiviert. Als Brenngas für diese Versuche dient Wasserstoff.

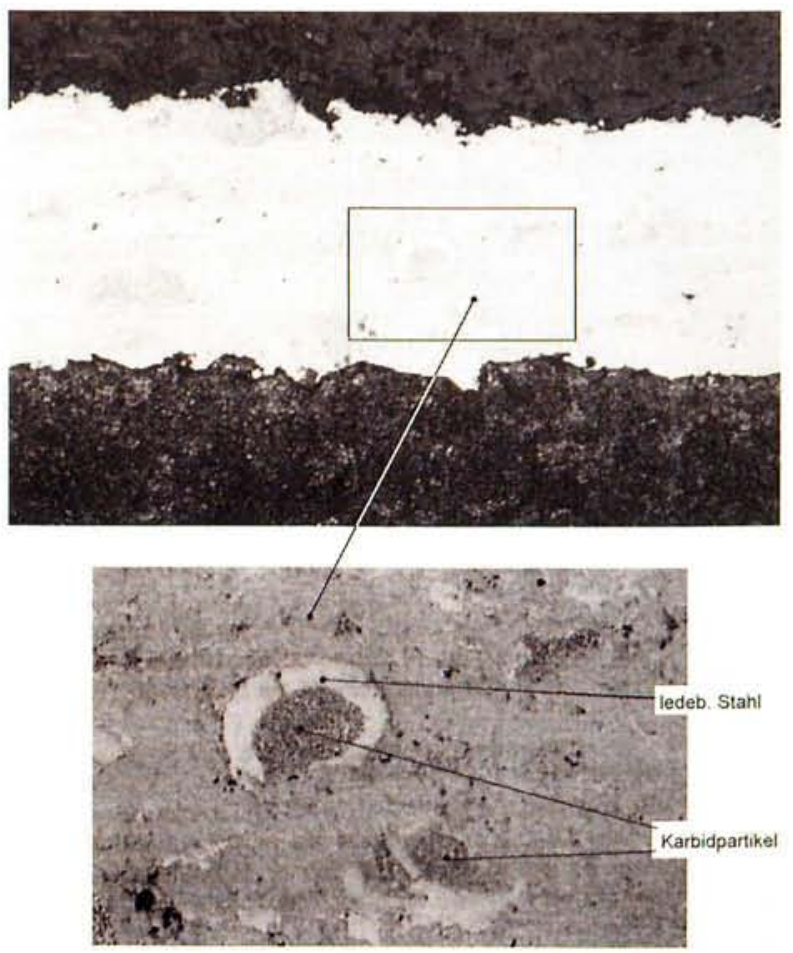

Bild 1: Querschliff, unterschiedliche Kornfraktion, 200:1 und 500:1

Um konkrete Aussagen über die Schichtstruktur und die Verteilung der einzelnen Schichtphasen treffen zu können, wurden metallographische Querschliffe untersucht. Dazu wurden die beschichteten Proben getrennt und präpariert. Diese Präparationsschritte waren für die jeweiligen Schichtwerkstoffe unterschiedlich.

Die Schichtdicken sind durch die jeweils unterschiedliche Pulverförderrate und den parameterabhängigen Wirkungsgrad verschieden.

Es ist erkennbar, daß alle Schichten vom Aufbau her nahezu identisch sind. Sie weisen alle eine sehr geringe Porosität auf. Die im Querschliff (Bild 1) als graue Punkte erscheinende Phase der Schicht zeigt das Wolframkarbid.

Bei näherer Betrachtung ist erkennbar, daß diese Karbidpartikel von der helleren Phase, dem Kobalt, regelrecht ummantelt wird. Die Wolframkarbide sind über den gesamten Schichtquerschnitt gleichmäßig fein verteilt. Die als großes Korn erscheinende helle Phase stellt die eigentlich als Matrixzusatz bestimmte ledeburitische Stahllegierung dar. Da dieses Material eine zu große Kornfraktion aufweist, kann dieser Werkstoff seine Funktion, die darin bestand feine einzelne Chrom- und Eisenkarbide in die Schichtmatrix miteinzubinden, nicht erfuillen.

Anhand der durchgeführten Analyse ist erkennbar, daß sich in den grobkörnigen Phasen Chrom und Eisen in Verbindung mit Kohlenstoff befinden. 

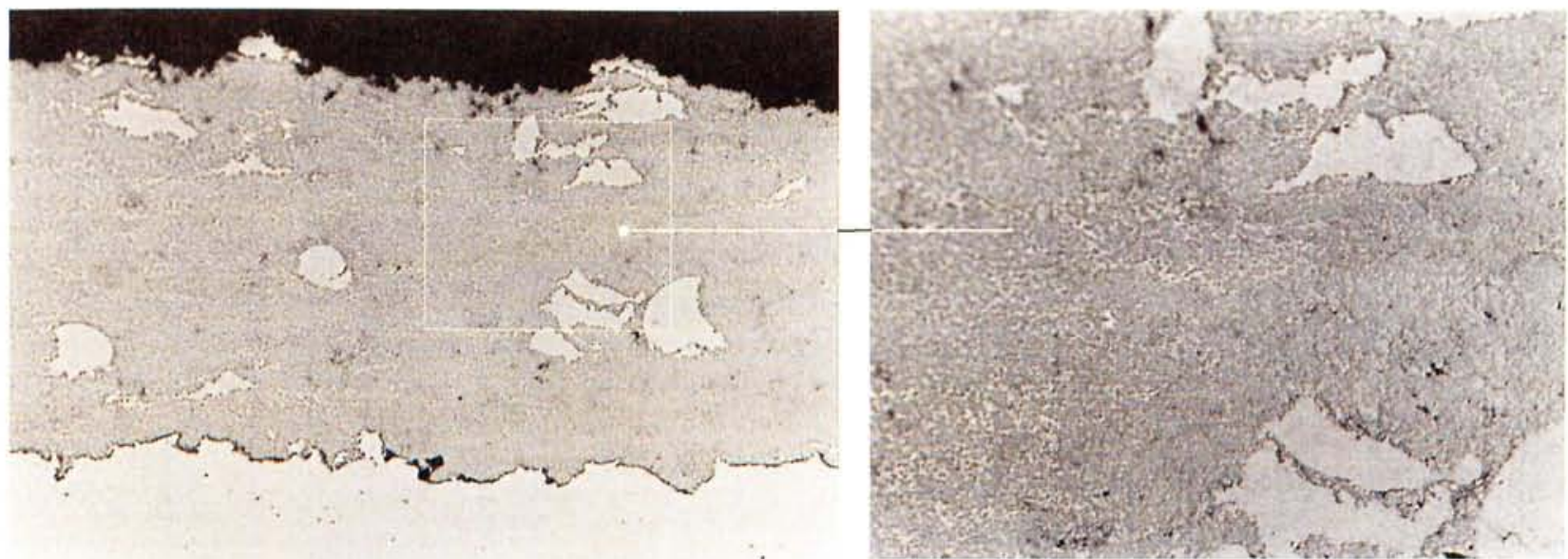

Bild 2: Querschliff des optimierten Spritzversuches, 200:1 und 500:1

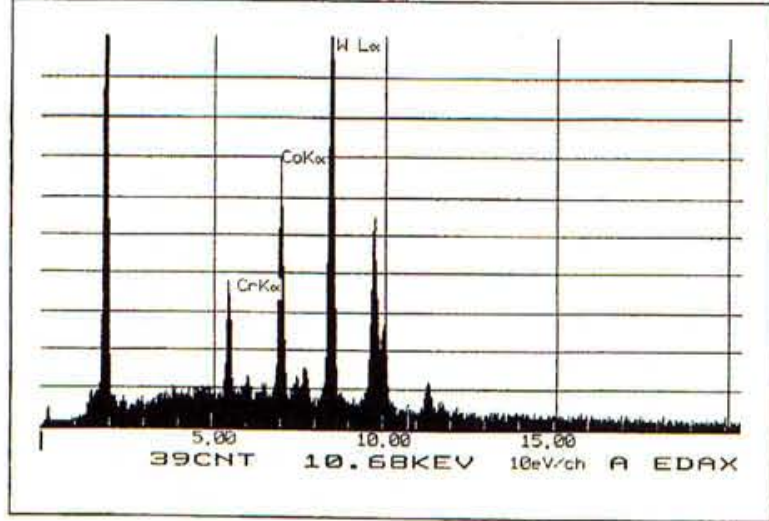

Bild 3: Analyse der hellen Phase

Als Ergebnis dieser Versuche muß festgestellt werden, daß das ledeburitische Stahlpulver mit dieser Kornfraktion schwierig zu verarbeiten ist. Um werkstoff- und spritztechnisch sinnvolle Ergebnisse zu erzielen, muß für den Matrixwerkstoff eine dem Amdry 5843-Pulver ähnliche Kornfraktion verwendet werden. Bei der Verwendung eines feineren ledeburitischen Spritzzusatzes ist eine bessere Vermischung von Eisenkarbiden und Chromkarbiden in der gesamten Matrix zur erwarten, was die Verschleiß- und Korrosionsbeständigkeit der Schicht erhöhen könnte.

Nach diesen Versuchen wurden die Spritzparameter optimiert und weitere Spritzversuche durchgeführt. Die ungünstige, zu hohe Kornfraktion der ledeburitischen Stahllegierung wurde dabei außer acht gelassen.

Durch die Analyse werden die prozentualen Massenanteile der einzelnen Schichtelemente vom Querschliff des optimierten Versuches ermittelt. Dabei wird die Verteilung der Elemente in der Schicht sowie die Zusammensetzung der unterschiedlichen Schichtphasen untersucht.

Das Bild (2) stellt einen Ausschnitt aus dem Ouerschliff des Versuches mit optimierten Parametern dar, an dem die prozentuale Verteilung der Massenanteile der Schicht insgesamt gemessen wird.

Die Analyse dieses Ausschnittes ergab, daß die Schicht einen durchschnittlichen Anteil von 56,64\% Eisen, $19,34 \%$ Chrom, $14,29 \%$ Wolfram und $9,73 \%$ Kobalt be-

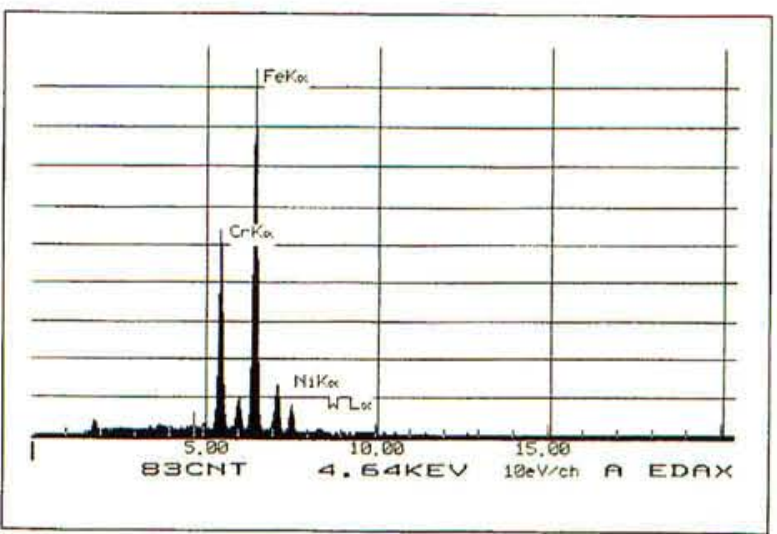

Bild 4: Analyse der dunklen Phase

sitzt. Die Elemente Kohlenstoff und Sauerstoff können aufgrund ihrer niedrigen Elektronenzahl mit dem zur Verfügung stehenden EDX-Systems nicht nachgewiesen werden, womit die Masseanteile der Karbide und Oxide nicht bestimmt werden können.

Das Bilder (3) und (4) zeigen das EDX-Analysediagramm mit der Verteilung der einzelnen Schichtbestandteile. Die Analyse der hellen Phase zeigt, daß es sich bei diesem Schichtbestandteil um das Hartstoffsystem handelt. Die Phase besteht aus $82,35 \%$ Wolfram, $13,46 \%$ Kobalt und $4,19 \%$ Chrom. Die Untersuchung der dunklen Phase ergab, daß es sich hierbei um den ledeburitischen Bestandteil der Schicht handelt. Diese Phase weist 66,08\% Eisen, $28,05 \%$ Chrom und $5,87 \%$ Nickel auf.

\section{Verschleißschutzschicht aus Siliziumkarbid in Verbindung mit einer metallischen Matrix}

Eine weitere Aufgabe in dieser Versuchsreihe besteht darin, mittels Hochgeschwindigkeitsflammspritzen zu untersuchen, ob ein neuartiges Hartstoffsystem, wie Siliziumkarbid in Verbindung mit einer metallischen Matrix, als Verschleißschutzschicht spritztechnisch herstellbar ist.

Da über die spritztechnische Verarbeitung von Siliziumkarbid mit dem HVOF nur unzureichende Kenntnisse existierten, wurden systematische Vorversuchsreihen durchgefuihrt. Die gewonnenen Erkenntnisse einer Versuchsreihe dienten als Basis für die weiteren Untersuchungen. 


\section{Eigenschaften von Siliziumkarbid und der verwendeten metallischen Matrix}

Das Hartstoffsystem Siliziumkarbid zeichnet sich durch einen niedrigen thermischen Ausdehnungskoeffizienten und eine sehr gute thermische Leitfähigkeit aus. Der Werkstoff besitzt eine große Härte, chemische Beständigkeit sowie Festigkeit bis $1350^{\circ} \mathrm{C}$. Mit Sauerstoff reagiert $\mathrm{SiC}$ bei hohen Temperaturen unter Bildung von $\mathrm{SiO}_{2}$ und $\mathrm{CO}$.

Für die hier durchgeführten Untersuchungen wurde ebenfalls, wie bei den Versuchen des Hartstoffsystem WC-Co-Cr mit der ledeburitischen Matrix, das $\mathrm{SiC}$ in Pulverform den Spritzprozeß zugefuihrt. Es standen verschiedene Pulverreinheiten, Partikelgrößen und -formen als kommerziell erhältliche Pulver zur Auswahl. Gewählt wurde eine runde Kornform.

Die Versuche sollten zeigen, ob die positiven Eigenschaften des $\mathrm{SiC}$ auch in thermisch gespritzten SiC-Schichten, die in einer metallischen Matrix eingebettet sind, zum Tragen kommen. Die Metallmatrix dient dazu, die einzelnen Karbidpartikel in die Schicht mit einzubinden und einen möglichst reinen, gleichmäßig verteilten und feinkörnigen Karbidanteil in der Schicht zu erzeugen. Beim Spritzvorgang sollen die SiC-Partikel erhalten bleiben, sich nicht zersetzen bzw. verdampfen. Siliziumkarbid besitzt keinen ausgeprägten Schmelzpunkt und neigt bei zu hoher Wärmezufuhr sofort zum Sublimieren.

Als Schichtmatrix für das Hartstoffsystem wird ein Spritzpulver mit der Bezeichnung „43F-NS“ gewählt. Hierbei handelt es sich um eine spezielle Nickel-ChromLegierung, die sich durch besondere Oxydations- und Korrosionsbeständigkeit auszeichnet. Der Matrixwerkstoff besteht aus $80 \%$ Nickel und $20 \%$ Chrom. Der Schmelzpunkt der Legierung liegt bei ca. $1400^{\circ} \mathrm{C}$. Bei der Wahl der Metallmatrix für das Siliziumkarbid muß außerdem darauf geachtet werden, daß beide Pulver ähnliche Korngrößen besitzen, da ansonsten Probleme bei der mechanischen Vermischung auftreten. Die Kornfraktion der gewählten metallischen Matrix liegt zwischen 10 und $53 \mathrm{~mm}$.

Nach mehreren Spritzversuchen, mit sich ändernden Spritzpulverzusammensetzungen, veränderter Flammentemperatur und geändertem Düseneinsatz, soll hier nur der letzte Spritzversuch dargestellt werden.

Für die Durchführung dieser Vorversuchsreihe sind aus den bisherig gewonnenen Erkenntnissen heraus eine Veränderung des Mischungsverhältnisses der Massenanteile und die Wahl einer feineren Kornfraktion des Spritzusatzes erforderlich. Es wurden an dieser Stelle zwei unterschiedliche Mischungsverhältnisse untersucht. Die gewählte Kornfraktion des Siliziumkarbides beträgt 15 bis $38 \mathrm{~mm}$, so daß wiederum das feinere Korn verwendet wurde. Eine weitere Veränderung ist die Senkung der Pulverförderrate von $50 \mathrm{~g} / \mathrm{min}$ auf $40 \mathrm{~g} / \mathrm{min}$, was der abrasiven Wirkung der karbidischen Anteile während des Spritzprozesses entgegenwirken soll.

Die weiteren Parametereinstellungen blieben gegenüber der Vorversuchsreihen unverändert.

\section{Auswertung der Versuchsreihe}

Die benutzten Parameter und die Ergebnisse sind in der Tabelle 1 festgehalten.

\begin{tabular}{|l|c|c|}
\hline $\begin{array}{l}\text { Spritzabstand }[\mathrm{mm}] \\
\begin{array}{l}\text { Mischungsverhältnis } \\
\text { (Matrix/Hartstoff) }\end{array}\end{array}$ & 260 & 260 \\
Spritzrate $[\mathrm{g} / \mathrm{min}]$ & 40 & 40 \\
Flammenverhältnis $\left(\mathrm{O}_{2} / \mathrm{C}_{3} \mathrm{H}_{8}\right)$ & 4,63 & 4,63 \\
Schritthöhe [mm] & 4 & 4 \\
Makrohärte [HR 15] & 80,7 & 83,2 \\
Porosität [\%] & $1-3$ & $1-3$ \\
Schichtdicke [ $\mu \mathrm{m}]$ & $100-165$ & $80-150$ \\
\hline Tabelle 1: Parameter und Ergebnisse &
\end{tabular}

Die Ergebnisse der beiden durchgefuihrten Versuche zeigen im Querschliff eine Schicht mit einem hohen gleichmäßig verteilten karbidischen Anteil. Die Schichten zeichnen sich durch eine geringen Porosität aus. Die Schichtdicke konnte aufgrund des größeren Matrixanteiles im Spritzzusatz erhöht werden.

Der Vergleich der Querschliffaufnahmen beider Versuche ergab, daß die Schicht des Versuches 9 einen hohen Karbidanteil aufweisen kann. Der Grund dafür ist das Mischungsverhältnis der Massenanteile. Die Pulvermischung des Versuches 9 weist einen Massenanteil des Siliziumkarbides von $80 \%$ auf, welches $10 \%$ mehr sind als der Anteil im Versuch 8. Dieser Unterschied scheint vermutlich, in Bezug auf das Erzielen eines hohen Karbidanteiles, eine entscheidende Rolle zu spielen. Dieses ist auch der Grund, daß der Versuch 9 eine in den bisherig durchgeführten Experimenten noch nicht erreichte Makrohärte von $83,2 \mathrm{HR}_{15 \mathrm{~N}}$ aufweisen kann.

Die Mikrohärte war jedoch gegenüber anderen Verschleißschutzschichten relativ gering. Die Ursache kann in der Größe und der Verteilung der Karbide liegen.

Zur Ermittlung der prozentualen Massenanteile der einzelnen Schichtelemente wurde vom Querschliff des Versuches 9 eine Analyse durchgefuihrt. Untersucht wurden zum einen die prozentuale Verteilung der Elemente insgesamt und zum anderen die Zusammensetzung der unterschiedlichen Phasen.

Das Bild (4) stellt einen Ausschnitt aus dem Querschliff des Versuches 9 dar, an dem die Verteilung der prozentualen Massenanteile der Schicht insgesamt gemessen wurde.

Das Ergebnis dieser Analyse belegt, daß die helle Phase die metallische Matrix darstellt. Sie besteht aus $74,83 \%$ Nickel und $25,17 \%$ Chrom. Die dunkle Phase beinhaltet $100 \%$ Siliziumkarbid. 

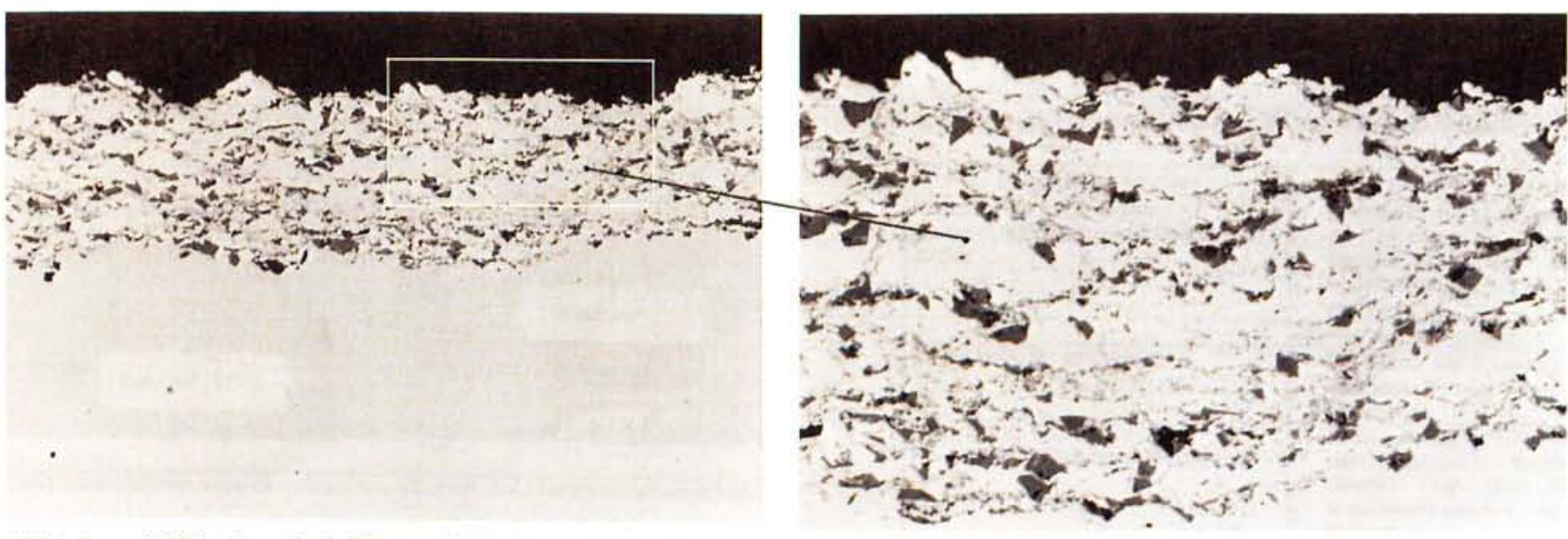

Bild 4: Querschliff des Versuchs 9, 200:1 und 500:1

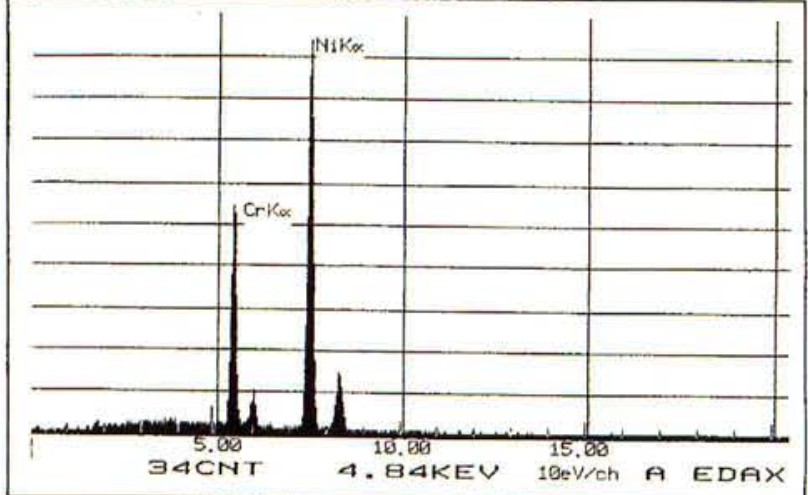

Bild 5: Analyse für die helle Phase

\section{Zusammenfassung der Ergebnisse}

Die Ergebnisse dieser Versuchsreihen zeigten, daß das Hartstoffsystem Siliziumkarbid mit der gewählten Nikkel-Chrom-Matrix durch das HVOF verarbeitbar ist. In den beiden Versuchen konnte nachgewiesen werden, daß eine Schicht mit geringer Porosität, homogener Struktur und hoher Makrohärte herstellbar ist.

Um die hergestellten Spritzschichten besser beurteilen zu können, müssen Verschleißuntersuchungen an den beschichteten Oberflächen durchgeführt werden.

Von der wirtschaftlichen Seite betrachtet, stellt das Siliziumkarbid eine sehr preisgünstige Alternative zu den alt bekannten karbidischen Spritzzusätzen dar. Der Preis des Siliziumkarbides hängt aber im wesentlichen von dem Herstellungsaufwand des Spritzzusatzes ab. So steigen beispielsweise die Kosten, wenn eine feinere Kornfraktion und ein höherer Reinheitsgrad des Siliziumkarbidpulvers verwendet wird.

Die Untersuchungen zeigen, welche umfangreichen Versuche und Analysen notwendig sind, um neuartige Spritzschichten zu erproben und zu optimieren.

Thermische Beschichtungen unterliegen während des Spritzprozesses vielen Einflußfaktoren, was eine Optimierung der Schicht sehr schwierig macht. Die Benutzung einer statistischen Versuchsplanungsmethode ist für diese Optimierung unerläßlich.

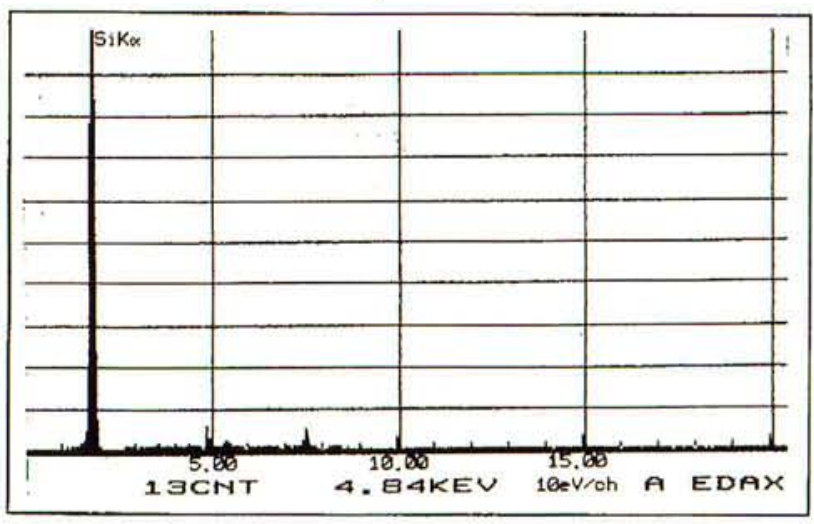

Bild 6: Analyse für die dunkle Phase

\section{Literaturverzeichnis}

[1] Schult, M., „Untersuchung und Optimierung der Parameter fuir das Hochgeschwindigkeitsflammenspritzen von karbidpartikelverstärkten Verschleißschutzschichten“, Diplomarbeit - TFH Wildau, 1996

[2] Heinrich, P., Thermisches Spritzen, Sonderdruck der Fa. Linde AG, Metall-Verlag GmbH, 1992

[3] Steffens, H.-D., Thermisches Spritzen, Universität Dortmund-Workshop, 1994

[4] Autorengruppe, Tagung 3. Kolloquium-Hochgeschwindigkeitsflammspritzen, Ingolstadt 1994

\section{Verfasser}

Prof. Dipl.-Ing. Armin Abel

Technische Fachhochschule Wildau

Fachbereich Ingenieurwesen/Wirtschaftsingenieurwesen Tel. (0 33 75) 507-104 